\title{
Multiple-phase trajectory optimization for formation flight in civil aviation
}

\author{
Sander Hartjes ${ }^{1} \cdot$ Marco E. G. van Hellenberg Hubar ${ }^{1} \cdot$ Hendrikus G. Visser $^{1}$
}

Received: 1 June 2017 / Revised: 5 September 2018 / Accepted: 17 September 2018 / Published online: 21 September 2018

(C) The Author(s) 2018

\begin{abstract}
In this paper a tool is developed that optimizes the trajectories of multiple airliners that seek to join in formation to minimize overall fuel consumption or direct operating cost. The developed optimization framework relies on optimal control theory to solve the multiple-phase optimization problem associated to flight formation assembly. A reduced-order point-mass formulation is employed for modelling of the aircraft dynamics within an extended flight formation, and of the solo flight legs that connect the flight formation to the origin and destination airports. When in formation, a discount factor is applied to simulate a reduction in the induced drag of the trailing aircraft. Using the developed tool a case study has been conducted pertaining to the assembly of two-aircraft formation flights across the North-Atlantic. Results are presented to illustrate the synthesis of the formation trajectories and to demonstrate the potential for reducing fuel and operating cost. The results of the various numerical experiments show that formation flight can lead to significant reductions in fuel consumption compared to flying solo, even when the original trip times are maintained. Additionally, the results clearly reveal how the performance and the characteristics of the flight formation mission—notably the location of rendezvous and splitting points—are affected when one aircraft seeking to join the formation suffers a departure delay.
\end{abstract}

Keywords Flight formations - Trajectory optimization · Fuel burn · DOC

\section{Introduction}

The potential to significantly reduce aircraft induced drag in extended formation flight has been clearly demonstrated in a range of numerical and experimental studies [1-4]. In contrast to close formation flight, in an extended formation aircraft are longitudinally separated by $5-40$ wingspans. The studies presented in [1-4] all agree that extended formation flight has the potential to significantly reduce fuel and operating costs of long-haul airline operations.

One of the key areas on which past research on (extended) formation flight has focused is the planning and organization of flight formations on a network-wide scale [3-10]. Most studies addressing the problems of global routing and assignment typically rely on a bi-level (or two-stage) approach. At the first level/stage, the routing/mission design problem is

Hendrikus G. Visser

h.g.visser@tudelft.nl

Faculty of Aerospace Engineering, Delft University of Technology, P.O. Box 5058, 2600 GB Delft, The Netherlands considered. In the routing/mission design problem, a candidate set of two or three long-haul origin/destination flights is considered that might join in, respectively, a two or three-aircraft formation. The routing/mission problem deals with locating the rendezvous and splitting points for the flights involved and with scheduling the associated altitude/speed profiles such that the overall mission (fuel) cost is minimized. Evidently, an optimized flight formation option is only accepted as a mission possibility if it saves (fuel) cost relative to flying the aircraft solo from origin to destination. The second level/ stage then seeks to optimize the network by selecting the best subset of formation and solo missions given the complete set of all possible combinations of individually optimized formation and solo missions. It is noted that the second level/stage is highly combinatorial in nature, as the number of options to join flights in formations grows dramatically with the number of flights in the network. Given the fact that for a network of realistic size, a huge number of formation options needs to be evaluated, it is clear that for the first stage mission design problem a computationally efficient optimization approach is warranted. For this reason, simplified, low fidelity aircraft models have typically been adopted to rapidly assess the (fuel) 
cost and trip times for the formations missions. Aircraft performance models employed for this purpose range from the Breguet-range equation at constant altitude evaluated along a geometrically constructed route [6], to a parameterized aircraft performance model in a three-dimensional space, featuring a limited number of design parameters [7]. Although computationally efficient, these low fidelity models do not provide very accurate results and are not quite capable of dealing with real-world complexities such as wind/weather influences and schedule delays.

In this study, a higher-fidelity mission design optimization model is developed that is capable of dealing with these real-world complexities at a reasonable computational cost. More specifically, the study presented in this paper deals with the development of a multi-phase optimal control framework that supports the synthesis of two-aircraft long-haul formation missions, using a point-mass dynamic model formulation. For each of the two flights considered, the origin/destination city-pair is given, along with the scheduled departure time. The primary reason for resorting to a multi-phase trajectory optimization formulation for the mission design problem is that it allows the concurrent optimization of the single two-aircraft formation flight leg, and the four solo flights legs connecting, respectively, the two origin airports to the rendezvous point and the two destination airports to the splitting point of the formation. It is noted that in the adopted multi-phase set-up both aircraft are forced to join in formation, even when flying the city-pair trajectories in solo flight might be more favourable. For this reason, the optimization framework also assesses the optimal performance of the two city-pair (great circle) trajectories flown solo, to enable a comparison. The potential (fuel) cost improvements realized by formation flight are then assessed against the cost of the combined solo flights.

In a case study, involving the Transatlantic crossing of two Boeing B747-400 aircraft, the developed multiple-phase optimization tool is deployed to optimize the trajectories of the aircraft that join in formation and experiments are conducted to investigate what the general characteristics and the potential benefits of formation flight are. In particular, it is explored whether fuel savings can still be obtained if no increase in trip time is permitted relative to flying solo. Additionally, it is assessed to what extent (fuel) cost benefits can still be achieved when one of the participating aircraft suffers a departure delay.

\section{Trajectory optimization formulation}

\subsection{Flight path modelling}

In this study we seek to optimize a two-aircraft flight formation for minimum cost or fuel burn. A basic scenario is considered in which two independent long-haul flights are assumed to depart from closely located origin airports towards two closely located destination airports, flying minimum (fuel) cost great circle trajectories. It is assumed in this scenario that the considered flights can join in formation with modest deviations from their original solo paths. Additionally, a no-wind condition is assumed in the baseline scenario. Another major assumption relates to scheduled departure times of the two flights considered. Essentially, it is assumed in the baseline scenario that the departure times of the two flights are perfectly synchronised to achieve minimum (fuel) cost. In a next stage, the influence of departure times that are not perfectly synchronised is explored.

In Fig. 1a, the basic routing problem in the baseline scenario is sketched, assuming two flights, labelled A and B, respectively. Both the solo and formation flight missions are indicated in Fig. 1a. The two independent solo flights depart at times $t_{0, \mathrm{~A}}$ and $t_{0, \mathrm{~B}}$, respectively, arriving at their destinations at $t_{\mathrm{f}, \mathrm{A}}$ and $t_{\mathrm{f}, \mathrm{B}}$, respectively. The formation flight is modelled as a multi-phase problem, comprising five stages. The first two stages connect the departing flights to the rendezvous point, where the formation stage is started (stage 3). Stages 4 and 5 connect flights A and B to their respective destination airports, after the formation has split up. The initial times for stages 1 and 2 are, respectively, $t_{0,1}=t_{0, \mathrm{~A}}$ and $t_{0,2}=t_{0, \mathrm{~B}}$. For rendezvous it is required that at the end of stages 1 and 2, $t_{\mathrm{f}, 1}=t_{\mathrm{f}, 2}=t_{\text {rendezvous }}=t_{0,3}$. It is noted that in the baseline scenario pertaining to the two-aircraft formation only the time instance $t_{\text {rendezvous }}$ is specified a priori. The ideal departure times $t_{0,1}$ and $t_{0,2}$ for flights $\mathrm{A}$ and $\mathrm{B}$ joining in formation are then determined in the optimization process. The imposed departure times $t_{0, \mathrm{~A}}$ and $t_{0, \mathrm{~B}}$ for the single

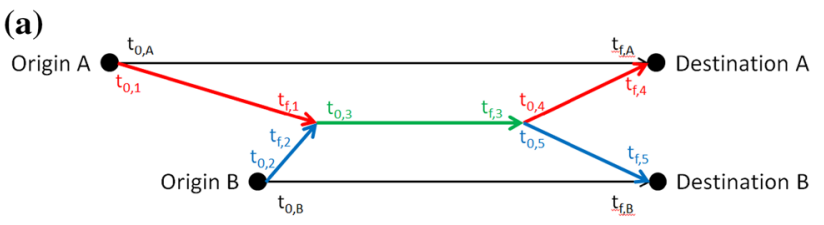

(b)

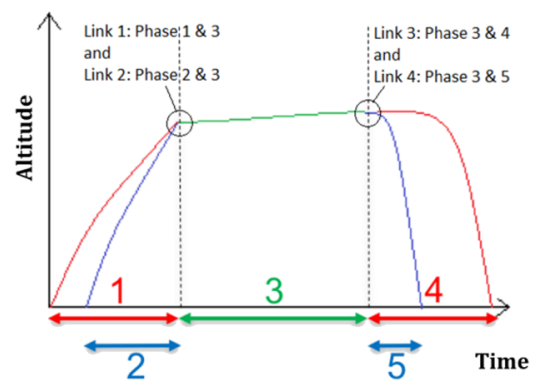

Fig. 1 A schematic representation of the five-phase assembly of a two-aircraft formation 
flights are set equal to the values $t_{0,1}$ and $t_{0,2}$ a posteriori, to allow a reference solution based on solo flights.

Figure $1 \mathrm{~b}$ shows the five-phase solution schematically in the vertical plane, highlighting the required phase linking conditions. As the aircraft will not join in formation during the take-off and approach phases of flight, these segments will remain the same for aircraft that will or will not join in formation. For this reason, the take-off and landing phases are not considered in this study and, the initial and final points of the mission are, respectively, the entry and exit points of the Terminal Manoeuvring Area (TMA), located at an altitude of 10,000 ft AGL.

\subsection{Equations of motion}

The system of differential equations of motion employed in this study essentially describes the movement of two variable-weight point masses in a three-dimensional space. The employed system model is somewhat simplified in the sense that equilibrium of forces normal to the flight path is assumed (i.e., a so-called intermediate point-mass model is employed [11]) for both aircraft, flying either solo or in formation. The resulting equations of motion for the solo flight legs (flights A and B), as well as for the lead aircraft in the formation flight leg can be written as:

$\dot{\phi}=\frac{V \cos \gamma \sin \chi+V_{W_{\mathrm{N}}}}{R_{\mathrm{E}}+z}$

$\dot{\lambda}=\frac{V \cos \gamma \cos \chi+V_{W_{\mathrm{E}}}}{\left(R_{\mathrm{E}}+z\right) \cos \phi}$

$\dot{z}=V \sin \gamma$

$\dot{V}=\frac{g_{0}(T-D)}{W}-g_{0} \sin \gamma$

$\dot{\chi}=\frac{g_{0}}{V \cos \gamma} \frac{L \sin \mu}{W}$

$\dot{W}=-F_{\mathrm{c}}$,

where $\phi$ is the latitude, $\lambda$ is the longitude, $z$ is the altitude, $R_{\mathrm{E}}$ is the Earth radius, $V$ is the airspeed, $\gamma$ is the flight path angle, $\chi$ is the heading angle, $W$ is the aircraft gross weight, $g_{0}$ is the gravitational acceleration, $T$ is the thrust, $D$ is the drag, $\mu$ is the bank angle and $F_{\mathrm{c}}$ is the fuel flow. The fuel flow $F_{\mathrm{c}}$ is a function of altitude, velocity and thrust, i.e., $F_{\mathrm{c}}$ $=F_{\mathrm{c}}(\mathrm{z}, V, T)$. Furthermore, $V_{W_{\mathrm{N}}}$ and $V_{W_{\mathrm{E}}}$ are components of the wind velocity in North-South direction, and East-West direction, respectively. However, in the baseline scenario the wind components are ignored. The intermediate point-mass model comprises six state variables (the left-hand side in the equations above) and three control variables: the engine control setting $\eta$, the aerodynamic roll angle $\mu$ and the flight path angle $\gamma$. The engine control setting variable $\eta$, which is subject to the constraint $0 \leq \eta \leq 1$, has the following effect on the thrust of the aircraft:

$\mathrm{T}=\left(\mathrm{T}_{\max }-\mathrm{T}_{\min }\right) \eta+\mathrm{T}_{\min }$,

where $T_{\max }(h, V)$ and $T_{\min }(h, V)$ are the maximum and idle thrust of the engine, respectively. The aerodynamic forces are the usual. For lift:

$\mathrm{L}=C_{\mathrm{L}} q S=C_{\mathrm{L}} \frac{1}{2} \rho V^{2} S$,

where $C_{\mathrm{L}}$ is the lift coefficient, $q$ is the dynamic pressure, $\rho$ is the air density and $S$ is the wing surface area. And for drag:

$\mathrm{D}=C_{\mathrm{D}}\left(C_{\mathrm{L}}\right) q S \Rightarrow D=D\left(z, V, C_{\mathrm{L}}\right)$,

where $C_{\mathrm{D}}$ is the drag coefficient for which a parabolic drag polar will be assumed:

$\mathrm{C}_{\mathrm{D}}=C_{\mathrm{D}_{0}}(M)+K(M) C_{\mathrm{L}}^{2}$,

which consists of two parts: the zero-lift drag component $C_{\mathrm{D}_{0}}$ and the induced drag component $K(M) C_{\mathrm{L}}^{2}$. Both drag coefficient components are dependent on the Mach number $M$.

For the trailing aircraft in the formation flight leg, a highly simplified system model is used. Essentially, the speed and altitude dynamics that govern the lead aircraft are also used to govern the trailing aircraft. As a result, the two flights A and B essentially operate as one entity in the formation leg. However, as the weight and the drag characteristics of the trailing aircraft in the formation are typically not the same as for the lead aircraft, the thrust level required for station keeping is different from the lead aircraft as well. The thrust level required for the trailing aircraft can be assessed from the specific energy rate balance:

$\dot{E}_{\text {lead }}=\dot{E}_{\text {trail }} \Rightarrow\left[\frac{V(T-D)}{W}\right]_{\text {lead }}=\left[\frac{V(T-D)}{W}\right]_{\text {trail }}$

And thus:

$T_{\text {trail }}=\left[\frac{(T-D)}{W}\right]_{\text {lead }} \cdot W_{\text {trail }}+D_{\text {trail }}$,

subject to: $0 \leqslant \eta_{\text {trail }}=\frac{\left(T_{\text {trail }}-T_{\min }\right)}{\left(T_{\max }-T_{\min }\right)} \leqslant 1$

It is noted that specific energy $E$ is defined as:

$\mathrm{E}=z+\frac{V^{2}}{2 g_{0}}$ 
The reduction in fuel consumption due to formation flight is a result of a reduction in induced drag for the trailing aircraft. To evoke this induced drag reduction, a formation flight induced drag reduction factor $\varepsilon$ is introduced in the parabolic drag polar of the trailing aircraft

$\mathrm{C}_{\mathrm{D}}=C_{\mathrm{D}_{0}}+(1-\varepsilon) K C_{\mathrm{L}}^{2}$,

A fairly wide range of (measured or calculated) reduction factors can be found in the literature. In this case study a fairly conservative value in induced drag reduction of $25 \%$ $(\varepsilon=0.25)$ is assumed for the trailing aircraft of a two-aircraft formation. The adopted value is based on the results reported in [8], for a weight ratio 1 between lead and trailing aircraft.

The aircraft performance model that has been considered in this study pertains to a Boeing B747-400 wide-body aircraft [12]. The same performance model is used for both aircraft in the formation (or in solo flight), excepting the induced drag reduction factor. The take-off weight considered for the two aircraft in a (solo or formation) mission is typically different, and largely depends on the specified Origin/Destination stage length.

\subsection{Optimization criteria}

The primary goal of flight formation is to achieve an overall reduction in fuel burn. The performance index related to the combined fuel consumption of flights $\mathrm{A}$ and $\mathrm{B}$ is defined separately for the single flights and the two-aircraft formation flight missions. For the solo mission, the fuel burn criterion is defined as:

$J_{\text {fuel,single }}=\left[W_{\mathrm{A}}\left(t_{0, A}\right)-W_{\mathrm{A}}\left(t_{f, A}\right)\right]+\left[W_{\mathrm{B}}\left(t_{0, \mathrm{~B}}\right)-W_{\mathrm{B}}\left(t_{\mathrm{f}, \mathrm{B}}\right)\right]$

For the formation flight mission, the fuel burn criterion aggregates the fuel consumed in the five flight phases:

$J_{\text {fuel,formation }}=\sum_{i=1}^{5}\left[W_{i}\left(t_{0, i}\right)-W_{i}\left(t_{\mathrm{f}, i}\right)\right]$,

where $W_{i}$ is the weight of the aircraft A in flight phase $i=1,4$, of aircraft $\mathrm{B}$ in flight phase $i=2,5$, and of aircraft $\mathrm{A}+\mathrm{B}$ in flight phase $\mathrm{i}=3$.

Since the assembly of a flight formation generally requires the aircraft to make a detour, the trip times for the aircraft in a formation is typically larger than for the solo flights. This implies that, while flying formation might reduce the total fuel cost, it does not necessarily result in a lower direct operating cost (DOC), which is a combination of both time and fuel cost. To be able to allow a tradeoff between time and fuel cost in formation flight, a second objective function is defined, which is essentially a weighted composite of mission time and fuel criteria:
$J_{\text {DOC,formation }}=\alpha \sum_{i=1}^{n_{\mathrm{a}}}\left[t_{\mathrm{f}, i}-t_{0, i}\right]+(1-\alpha) \cdot \sum_{i=1}^{n_{\mathrm{a}}}\left[W_{i}\left(t_{0, i}\right)-W_{i}\left(t_{\mathrm{f}, i}\right)\right]$,

where $\alpha$ is an interpolation parameter $0 \leq \alpha \leq 1$, that can be selected to enable a shift in emphasis in the optimization process between mission time and mission fuel. In the case study conducted herein, the parameter $\alpha$ is systematically varied to allow the construction of a Pareto-front.

\subsection{Constraints, staging and boundary conditions}

The multi-phase formulation for the formation flight mission is subject to a range of boundary conditions and path and control constraints. At the initial times for both flights A and B, the position coordinates (latitude, longitude), altitude, speed and heading are specified. The same state variables are also fixed at the final times. It is recalled that in the baseline scenario, neither the initial times nor the final times are specified. Only the time at which the rendezvous takes place is fixed upfront. In the subsequent delay scenario, the initial times established in the baseline scenario are then fixed as boundary conditions, whilst removing the boundary condition related to the fixed rendezvous time. In this scenario, departure delay for one of the flights can be introduced by merely shifting the fixed initial time of the flight concerned.

The most complex set of constraints/boundary conditions relates to the initial and final weights of flights A and B. To minimize fuel consumption, a flight should land with a minimum amount of fuel remaining in the tanks. In normal circumstances, aircraft take enough fuel on board for the planned trip, plus a reserve. This reserve usually consists of contingency fuel and a final reserve fuel. The final reserve fuel is the minimum fuel required to fly for $30-45 \mathrm{~min}$ at $1500 \mathrm{ft}$ at holding speed above the destination or alternate aerodrome. Contingency fuel is carried to account for additional fuel consumption during the scheduled flight caused by, e.g., wind or ATM restrictions. In general this is around $5 \%$ of the trip fuel. Aircraft that join in formation will have to deviate from their optimal solo routes, resulting in an increase of the distance covered. In addition, the aircraft must also take into account that the other aircraft with which it intends to join, might not show-up (due to technical problems, delay, etc.). This implies that an aircraft should carry enough fuel to fly the detour distance solo. To enforce this, a constraint is introduced in the formulation that fixes the final weight of the aircraft. For aircraft flying a solo mission, the final weight of the aircraft is the sum of the Operational Empty Weight (OEW), the payload ( $\left.W_{\text {payload }}\right)$ and a fuel reserve of $5 \%$ of the total fuel capacity $\left(W_{\mathrm{FC}}\right)$ :

$W_{i}\left(t_{\mathrm{f}, i}\right)=\mathrm{OEW}+W_{\text {payload }}+0.05 W_{\mathrm{FC}}, i=\mathrm{A}, \mathrm{B}$ 
With the final weight of the solo flight fixed, the unspecified initial weight of the aircraft will be established in the trajectory optimization process. Evidently, the maximum take-off weight and fuel tank capacity constraints are taken into consideration. The initial/final weights of the lead aircraft in a formation are established in a fashion similar to that for the solo flights. It is noted that the take-off weight of the lead aircraft in a formation is usually somewhat larger than that of its corresponding solo flight (great circle route), due the fact that the lead aircraft needs to cover a larger distance to join the formation, but does not enjoy an induced drag reduction.

For the trailing aircraft in a formation, the assessment is more complicated. Indeed, if the trajectory of the trailing aircraft would be optimized for the final weight given by Eq. (18), the initial weight that would result from the multi-phase trajectory optimization is too low. The reason for this is that in the multiphase trajectory optimization the trailing aircraft enjoys an induced drag reduction and associated fuel burn savings, but it will not benefit from this drag reduction when it is forced to fly the same trajectory solo due to contingency circumstances. To allow for this, an additional state variable is added to the dynamic model of the trailing aircraft which represents the "pseudo" weight of the trailing aircraft. The final value of the pseudo weight of the trailing aircraft is fixed following Eq. (18). The pseudo weight of the trailing aircraft evolves over time in the same way as the actual aircraft weight, except that in the fuel flow calculations the influence of drag reduction due to formation flight is ignored (evolution as in a solo flight). An additional constraint is then introduced in the multiphase formulation that equates the "actual" initial weight of the trailing aircraft with the "pseudo" initial weight of the trailing aircraft. This constraint ensures that the final weight of the trailing aircraft is optimized for a fixed initial weight that would allow the aircraft to fly the (longer) formation route solo in case the other aircraft does not show-up.

In a multi-phase formulation so-called staging conditions need to be included. Staging conditions are constraints that specify how the state at the end of a particular phase corresponds to the initial state in a subsequent phase. In the present formulation, the staging conditions are quite simple in the sense that the initial state of a particular phase is directly and fully connected to the terminal state of the preceding phase. In Fig. 1b a schematic representation is shown of the vertical flight paths covering the five phases. These five phases are connected by four staging conditions (links):

- Link 1: Connects phase 1 with phase 3

- Link 2: Connects phase 2 with phase 3

- Link 3: Connects phase 3 with phase 4

- Link 4: Connects phase 3 with phase 5
In the trajectory optimization formulation it is important to decide upfront which of the two aircraft is designated as the lead aircraft. In the present set-up, where two aircraft of the same type are considered, it is clear what the best choice is: the least heavy aircraft of the two is designated as the lead aircraft, as the heavy aircraft can benefit relative more from an induced drag reduction. Indeed, as shown in [10], the lift-induced drag $D_{\mathrm{i}}$ in straight and level flight is proportional to weight squared:

$D_{i}=(1-\varepsilon) K \frac{W^{2}}{\frac{1}{2} \rho V^{2} S}$

and therefore, the induced drag for the heavier aircraft will be larger than for the lighter aircraft, for a given value of $\varepsilon$. The fact that the heavier aircraft should be leading in a formation is confirmed in the analytic study on cruise range in formation flight presented in [13]. However, the study reported in [9] arrives at a somewhat different conclusion about the preferred order, by showing that a formation is more likely to produce a fuel saving benefit if the weight of the leader is higher than that of the follower. In [9], the aerodynamic interactions within the formation are based on a more refined aerodynamic model that differs from that employed in this study, where essentially the model developed in [8] is implemented.

\section{Trajectory optimization framework}

The numerical trajectory optimization approach implemented in the optimization framework is based on a pseudospectral multiple-phase optimal control method. More specifically, a trajectory optimization tool named "GPOPS" has been implemented. GPOPS stands for "General Pseudospectral OPtimal Control Software" and is a MatLab-based general purpose software for solving multiple-phase optimal control problems using pseudospectral methods [14]; it does so using the Radau pseudospectral method [15]. It uses the technique of collocation at Legendre-Gauss points to transcribe the trajectory optimization problem to a nonlinear programming problem (NLP). GPOPS is used in combination with Interval Laboratory ("IntLab") which is a third party automatic differentiator, and with the nonlinear programming solver Sparse Nonlinear OPTimizer ("SNOPT").

For a two-ship formation problem, the typical execution time at a standard laptop CPU is around 1-10 min, depending on the grid coarseness and the quality of the initial guess. The transcribed NLP problems may comprise up to 12,500 constraints and 10,000 nonlinear decision variables. 


\section{Case study}

In a case study several numerical experiments have been performed to investigate the benefits of formation flight and the effect of schedule delays on the formation. Unless stated otherwise, in the experiments the trajectories are optimized for minimum fuel and aircraft $\mathrm{A}$ is the trailing aircraft. The aircraft are assumed to operate in a standard atmosphere in a no-wind condition.

\subsection{Baseline scenario}

In the baseline scenario the following two flights join in formation:

1. Aircraft A: A B744 from London to Atlanta.

2. Aircraft B: A B744 from Madrid to New York City.

The resulting horizontal flight paths are presented in Fig. 2 and the vertical flight paths, velocity profiles and specific energy profiles are presented in Fig. $3 \mathrm{a}-\mathrm{c}$, respectively.

When looking at the results, one of the first things that could be noticed are the rapid transitions in the altitude and the velocity variables near the joining point. Indeed, in a formation leg the two aircraft fly at a slightly higher altitude and a slightly lower speed than in single flight. This can be explained by the fact that the cruise condition for best specific range is at a somewhat lower speed relative to flying solo due to the lower induced drag of the trailing aircraft in formation. The reduction in induced drag leads to a lower total drag and a shift in the dynamic pressure at which the minimum total drag is obtained. At a given altitude, the

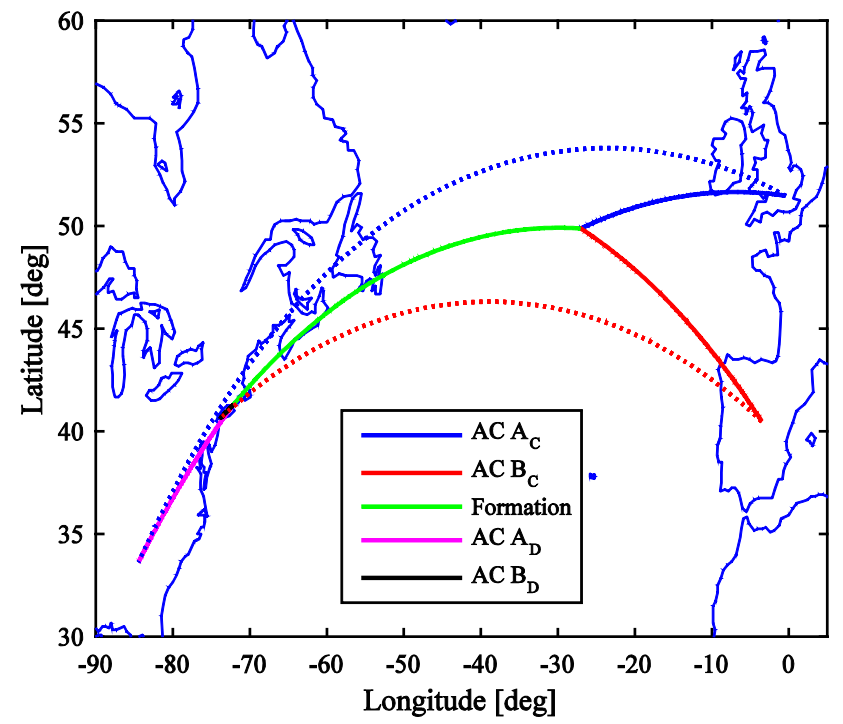

Fig. 2 Formation ground tracks for the baseline scenario
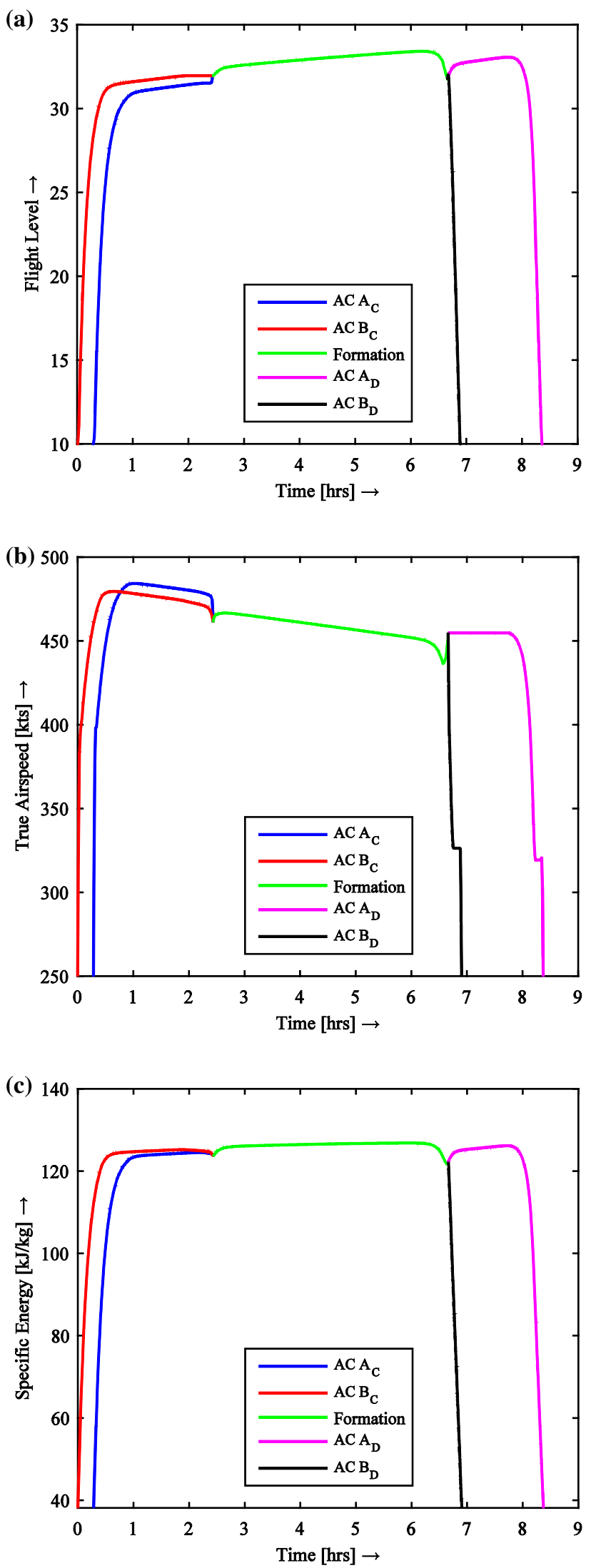

Fig. 3 Formation trajectory results for the baseline scenario (vertical plane) 
minimum total drag and even more relevant from a specific range perspective, the drag-to-speed ratio - is now achieved at a lower speed and therefore, the aircraft in formation slow down. Figure 4 illustrates this shift in optimal speed resulting from an induced drag reduction for the trailing aircraft.

The trajectory behaviour at the splitting point of the formation is perhaps even more surprising. In particular, it can be observed that the formation actually descents (both in terms of altitude and specific energy) before it splits-up. It is noted that the leading aircraft reduces its engine control setting $\eta$ in this early descent to about $70 \%$, but the lead aircraft requires a somewhat lower thrust setting as it is subject to a relatively lower drag. After splitting, aircraft A (the previously trailing aircraft) actually starts to climb again to the optimal cruise conditions for solo flight (and thus to a higher cruise speed than in formation flight). This (energy) climb requires additional thrust, and thus a higher fuel flow. Apparently, the additional fuel burn resulting from this late climb is offset by the fuel reduction in the early descent and flying in formation for a longer period of time. These peculiar "dents" in the energy histories occurred in all conducted numerical experiments to some extent, including those featuring different aircraft types. Numerical experiments in which the occurrence of these dents is precluded bear out that the mission fuel is indeed (modestly) increased in those

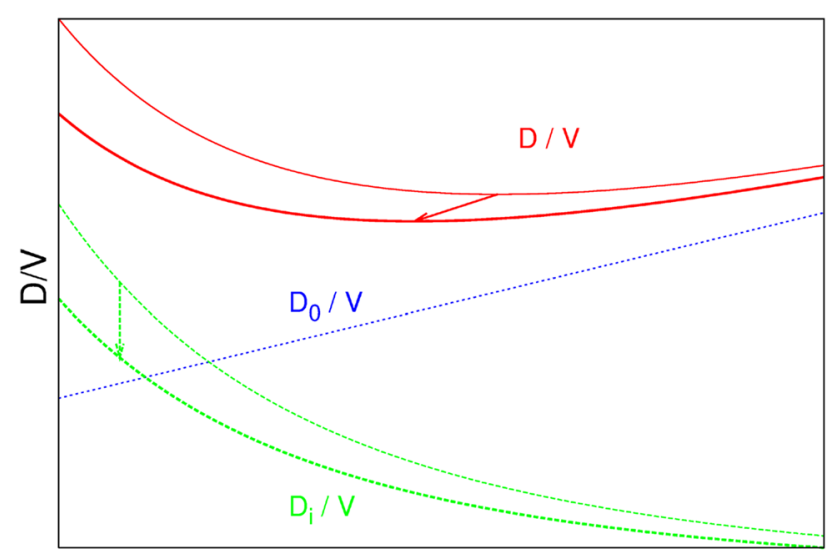

Airspeed

Fig. 4 Shift in optimal speed for minimum drag-to-speed ratio resulting from a reduction in lift-induced drag for a trailing aircraft in a flight formation cases. However, from an operational perspective, these constrained trajectories might actually still be preferred.

The main results for the baseline case are summarized in Table 1. Although the fuel consumption of the lead aircraft (B) increases compared to the solo flight, the overall fuel consumption in the formation mission decreases with approximately $1.8 \%$.

\subsection{Departure delay scenario}

In the baseline scenario the lowest total fuel consumption is obtained when aircraft A departs $1008 \mathrm{~s}$ later than aircraft B. So, for this set of flights the optimal initial times are $t_{0, \mathrm{~A}}$ $=1008 \mathrm{~s}(0.28 \mathrm{~h})$ and $t_{0, \mathrm{~B}}=0$. In the departure delay scenario, the departure times of both aircraft are fixed (whilst removing the fixed rendezvous time constraint), but now a departure delay of $30 \mathrm{~min}$ is assigned to aircraft $\mathrm{B}$, relative to the baseline scenario. Since in this delay scenario, aircraft A is the first one to depart, its departure time $t_{0, \mathrm{~A}}$ is set to zero. Hence in this scenario we consider:

1. Aircraft A: A B744 from London to Atlanta with initial time $t_{0, \mathrm{~A}}=0 \mathrm{~s}$.

2. Aircraft B: A B744 from Madrid to New York with initial time $t_{0, \mathrm{~B}}=792 \mathrm{~s}$.

The formation mission results for the departure delay scenario are summarized in Table 2. In Table 2 the results for the corresponding solo flights are presented as well, to allow a comparison.

The results of the delay scenario reveal that the fuel burn for the formation mission with one delayed aircraft is still (about 1.2\%) lower than that for the corresponding solo flights, while the total flight time increased only marginally compared to the no-delay (baseline) case. However, while aircraft B suffered the delay, the consequences of the delay recovery are particularly felt by aircraft $\mathrm{A}$, with its flight time increasing appreciably compared to the flight time of its corresponding solo flight. The shorter flight time of aircraft B is partly due to the increase in velocity (see Fig. 5) and partly due to the route change (Fig. 6). Aircraft A has to absorb a time delay and it does so by flying at the maximum endurance speed during a large part of the climb and by stretching its flight path in such a way that the flight path of its formation partner (aircraft B) is reduced.
Table 1 Results for the baseline scenario

\begin{tabular}{|c|c|c|c|c|c|c|}
\hline \multirow[t]{2}{*}{ Results } & \multicolumn{3}{|l|}{ Solo flight } & \multicolumn{3}{|c|}{ Formation flight } \\
\hline & Aircraft A & Aircraft B & Total & Aircraft a & Aircraft b & Total \\
\hline Fuel (kg) & 79,093 & 66,239 & 145,332 & 75,530 & 67,238 & 142,768 \\
\hline Time (h) & 7.92 & 6.80 & 14.71 & 8.09 & 6.90 & 14.99 \\
\hline Distance $(\mathrm{km})$ & 6760 & 5760 & 12,521 & 6825 & 5835 & 12,660 \\
\hline
\end{tabular}


Table 2 Results for the delay scenario

\begin{tabular}{lllllllll}
\hline Results & \multicolumn{3}{l}{ Solo flight } & & & & \multicolumn{2}{l}{ Formation flight } \\
\cline { 2 - 3 } & Aircraft A & Aircraft B & Total & & Aircraft A & Aircraft B & Total \\
\hline Fuel (kg) & 79,093 & 66,239 & 145,332 & 76,696 & 66,867 & 143,563 \\
Time (hr) & 7.92 & 6.80 & 14.71 & 8.37 & 6.67 & 15.06 \\
Distance $(\mathrm{km})$ & 6760 & 5760 & 12,521 & & 6850 & 5790 & 12,640 \\
\hline
\end{tabular}

(a)

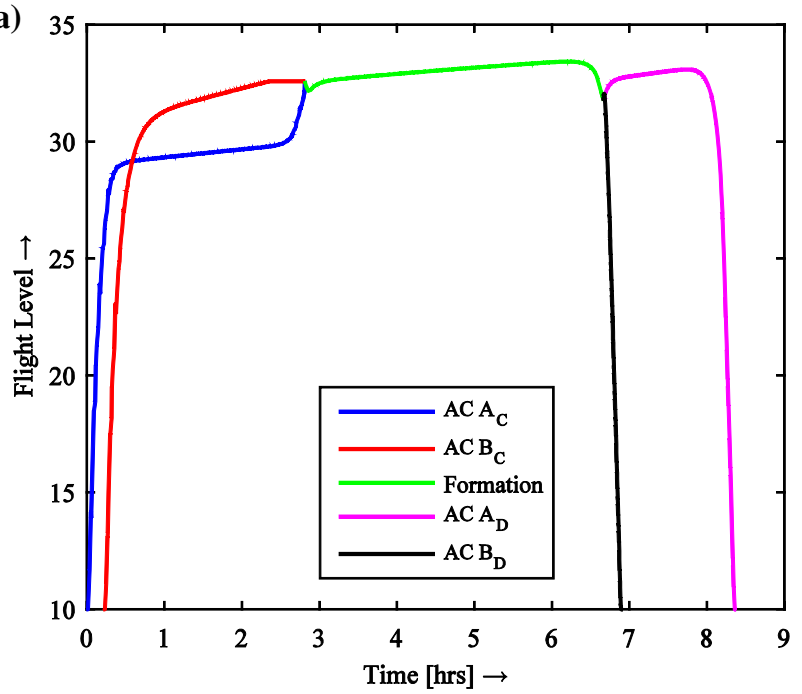

(b)

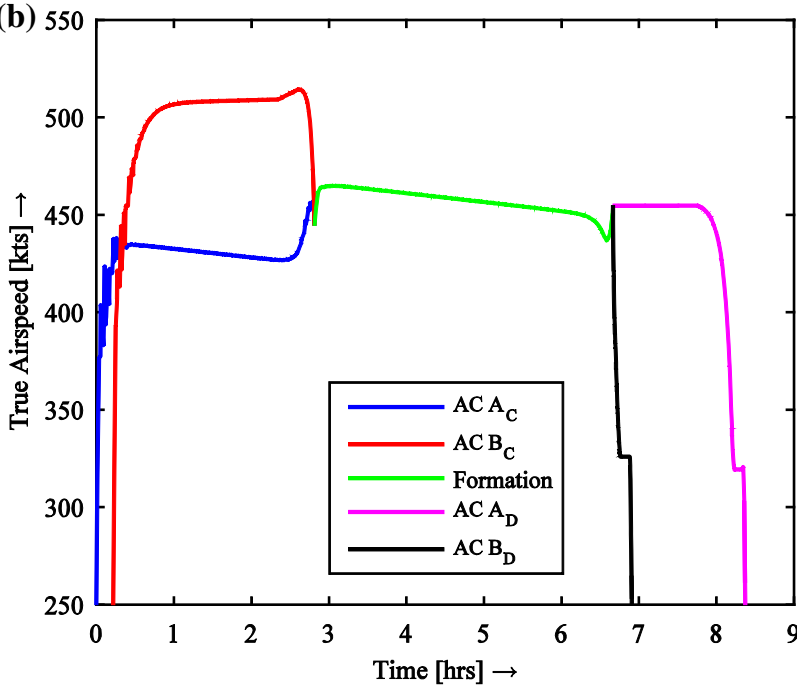

Fig. 5 Formation trajectory results for the delay scenario (vertical plane)

Figure 7 presents the resulting rendezvous points of several formations featuring one delayed aircraft. More specifically, Fig. 7 shows the displacement of the rendezvous point location when aircraft A or B are delayed by, respectively, 10,15 or $30 \mathrm{~min}$. It is clear that when one of the aircraft is delayed, the rendezvous point is displaced in both longitudinal and lateral direction.

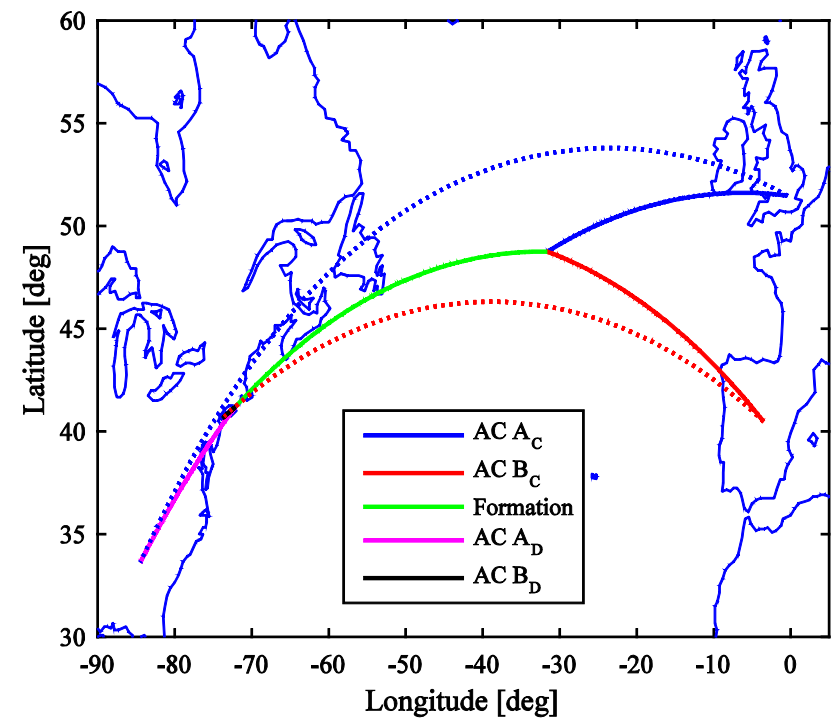

Fig. 6 Formation ground tracks for the delay scenario

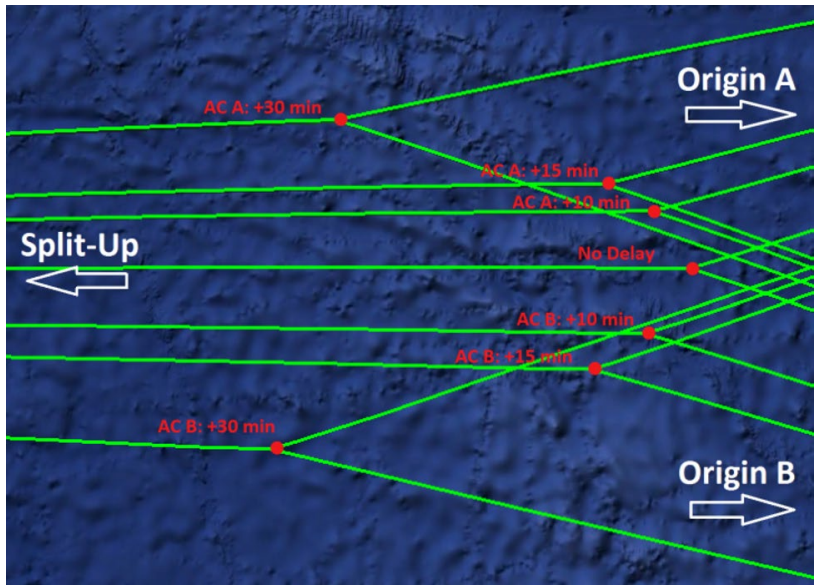

Fig. 7 Shift of rendezvous point when aircraft A or B is delayed relative to baseline scenario

\subsection{Direct operating cost scenario}

This scenario has been conceived to demonstrate what the effect on the performance of the aircraft in the formation is when in the trajectory optimization process the emphasis is gradually shifted from optimizing for fuel consumption to optimizing for flight time. A parametric study has been 


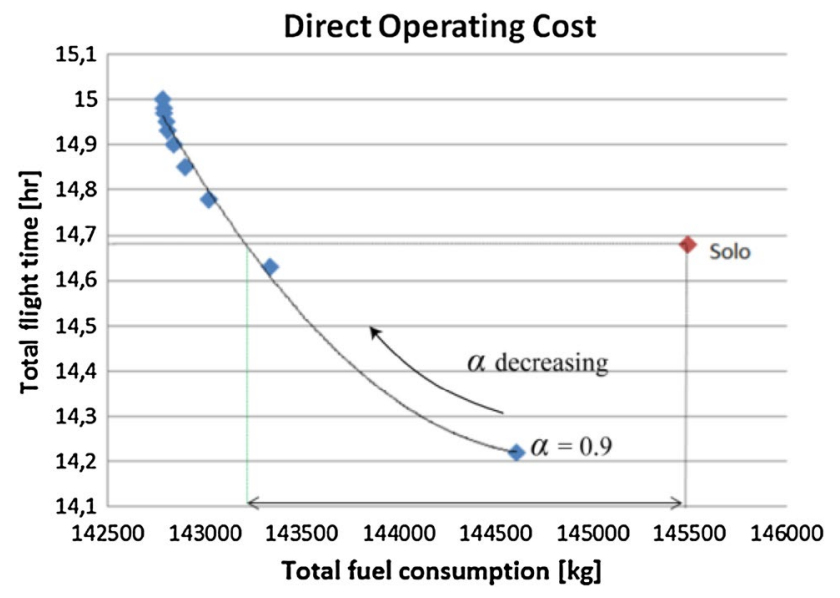

Fig. 8 Flight formation optimal fuel-time performances for various values of the weighting parameter $\alpha$

conducted in which the interpolation parameter $\alpha$ in the performance index (17) is systematically varied between zero and one. Since $\alpha=1$ corresponds to a cost function that purely considers flight time, it is left out of consideration. In this scenario, the same set-up is employed as in the baseline case, with the exception of the different performance index. In Fig. 8 the results of this parametric investigation are summarized.

The blue dots shown in Fig. 8 represent the results for the formation mission, where the dot in the upper left corner represents $\alpha=0$ (optimized for minimum total fuel consumption). When the value of $\alpha$ is increased (in steps of 0.1), Fig. 8 shows that the total flight time decreases while the total fuel burn increases. The combined results for the baseline (minimum fuel burn) solo flights are shown in Fig. 8 as well (represented by the red dot). It can be observed that the corresponding solo flights consume significantly more fuel compared to flying in formation, for any value of the parameter $\alpha$ in the considered range. When the value of the parameter $\alpha$ is set to $\approx 0.78$ in Eq. (17), the total flight time of the formation equals the total flight time of the corresponding solo flight, while the total fuel consumption is $2300 \mathrm{~kg}(1.58 \%)$ lower. This shows that, compared to flying solo, formation flight can lead to significant reduction in fuel burn, even when trip time is preserved. It should be kept in mind though, that this analysis is for this particular set of flights and aircraft type combination. For other origin and destination pairs or aircraft type combinations, the results might deviate from the results obtained in this scenario.

\section{Conclusions}

This paper has shown the possibility of optimizing the trajectories of aircraft that join in formation. The developed multiple-phase optimization tool is able to optimize trajectories of two or more aircraft for minimum total fuel consumption or Direct Operating Cost. Several numerical experiments have been performed in a case study to investigate the benefits of formation flight and the behaviour of the aircraft when joining in formation.

The results from the experiments show that formation flight can lead to significant reductions in fuel consumption, while not increasing the trip time. The results show that when aircraft fly in formation, the optimal airspeed decreases and the optimal altitude increases compared to the corresponding optimal solo flights.

Furthermore, the effects of departure delay on formations have been examined. This analysis revealed that in the cases that a departure delay was assigned to one of the participating formation partners, the location of the rendezvous point was shifted. In contrast to what has been often assumed in the literature, the rendezvous point location will not merely shift along the original (non-delayed) optimal route, but it will also deviate laterally (in the direction of the solo route of the delayed aircraft) such as to shorten the route of the delayed aircraft that seeks to join the formation.

Although in this paper only the optimization of twoaircraft formations was addressed, the developed optimization framework is capable of optimizing the trajectories of three-aircraft formations as well. For future research, it might be of interest to investigate the benefits of larger formations. Furthermore, the optimization in this research mainly focused on minimizing the total fuel burn or DOC, assuming a standard atmosphere and a no-wind condition. In future research, the benefits and characteristics of formation flight in real-world (weather/wind) conditions need to be explored. Finally, as optimizing the trajectories of more than two aircraft with the developed optimization framework proved to be very time-consuming, future research needs to be aimed at speeding up the computational process to render the optimization approach tractable in the context of bi-level planning of flight formations on a network scale.

Open Access This article is distributed under the terms of the Creative Commons Attribution 4.0 International License (http://creativeco mmons.org/licenses/by/4.0/), which permits unrestricted use, distribution, and reproduction in any medium, provided you give appropriate credit to the original author(s) and the source, provide a link to the Creative Commons license, and indicate if changes were made. 


\section{References}

1. Pahle, J., Berger, D., Venti, M., Duggan, C., Faber, J., Cardinal, K.: An Initial flight investigation of formation flight for drag reduction on the C-17 aircraft. In: AIAA Atmospheric Flight Mechanics Conference, 13-16 August 2012, Minneapolis, USA, p. 13 (2012)

2. Ning, S.A., Flanzer, T.C., Kroo, I.M.: Aerodynamic performance of extended formation flight. J. Aircr. 48(3), 855-865 (2011)

3. Veldhuis, L., Voskuijl, M., Fransen, B.: Formation flight-finetuning of theoretical performance prediction. In: AIAA Aerospace Sciences Meeting, 7-10 January 2013, Grapevine, Texas, USA, p. 17 (2013)

4. Ning, S.A., Kroo, I., Aftosmis, M.J., Nemec, M., Kless, J.E.: Extended formation flight at transonic speeds. J. Aircr. 51(5), 1501-1510 (2014)

5. Bower, G.C., Flanzer, T.C., Kroo, I.M.: Formation geometries and route optimization for commercial formation flight. In: AIAA Applied Aerodynamics Conference, 22-25 June 2009, San Antonio, Texas, USA, p. 18 (2009)

6. Kent, T., Richards, A.: Analytic approach to optimal routing for commercial formation flight. J. Guid. Control Dyn. 38(10), 1872 $1884(2015)$

7. Xu, J., Ning, S.A., Bower, G., Kroo, I.: Aircraft route optimization for formation flight. J. Aircr. 51(2), 490-501 (2014)

8. Xue, M., Hornby, G.S.: An analysis of the potential savings from using formation flight in the NAS. In: AIAA Guidance,
Navigation, and Control Conference, 13-16 August 2012, Minneapolis, USA, p. 12 (2012)

9. Marks, T., Gollnick, V.: Influence of aircraft type and order on fuel savings gained by two aircraft formations. In: Congress of the International Council of the Aeronautical Sciences, ICAS 20160592, 25-30 September 2016, Daejeon, South Korea (2016)

10. Verhagen, C.M.A., Visser, H.G., Santos, B.F.: A decentralized approach to formation flight routing of long-haul commercial flights. Proc. IMechE Part G J. Aerosp. Eng. (2018). https://doi. org/10.1177/0954410018791068

11. Visser, H.G.: Airplane performance optimisation. In: Encyclopaedia of aerospace engineering. Wiley, Oxford (2010)

12. Visser, H.G.: Environmentally optimized resolutions of in-trail separation conflicts for arrival flights. J. Aircr. 45(4), 1198-1205 (2008)

13. Voskuijl, M.: Cruise range in formation flight. J. Aircr. 54(6), 2184-2191 (2017)

14. Rao, A.V., Benson, D.A., Darby, C.L., Patterson, M., Francolin, C., Sanders, I., Huntington, G.T.: Algorithm 902: GPOPS, a MATLAB software for solving multiple-phase optimal control problems using the gauss pseudospectral method. ACM Trans. Math. Softw. 37(2), 22.1-22.39 (2010)

15. Francolin, C., Rao, A.V.: Direct trajectory optimization and costate estimation of state inequality path-constrained optimal control problems using a radau pseudospectral method. In: AIAA Guidance, Navigation, and Control Conference, 13-16 August 2012, Minneapolis, USA, p. 11 (2012) 